\title{
Worsening Muscle Weakness in Myasthenia Gravis Patient Suffering Dengue Infection
}

\author{
Hardjo Lugito NP ${ }^{1^{*}}$, Margaret ${ }^{2}$, Andree Kurniawan $^{1}$ and Merlyn Tjiang ${ }^{1}$ \\ ${ }^{1}$ Internal Medicine Department, Faculty of Medicine, Pelita Harapan University, Indonesia \\ ${ }^{2}$ Siloam Karawaci General Hospital, Indonesia
}

*Corresponding author: Nata Pratama Hardjo Lugito, Internal Medicine Department, Faculty of Medicine, Pelita Harapan University, Indonesia, Tel: 622154210130; Email: nata_pratama_hl@yahoo.com, nata.lugito@uph.edu

Rec date: Apr 10, 2014; Acc date: May 24, 2014; Pub date: May 27, 2014

Copyright: (c) 2014 Hardjo Lugito NP, et al. This is an open-access article distributed under the terms of the Creative Commons Attribution License, which permits unrestricted use, distribution, and reproduction in any medium, provided the original author and source are credited.

\begin{abstract}
The dengue infection is the second most common mosquito-borne disease affecting human beings. Dengue can manifest with a wide range of neurological features, which have been noted - depending on the clinical setting in $0.5-21 \%$ of patients with dengue admitted to hospital. Neuromuscular complication can manifest in muscle weakness, which can be found in forms of myalgia, myositis to rhabdomyolysis, Guillain - Barre syndrome and hypokalemia. The pathogenesis and also the role of host and virus in dengue neuromuscular complications were not clear. In this case report, a 36 year-old female MG patient suffered from dengue infection. The MG symptoms worsen and then improved along with the course of dengue. There was a possibility that muscle weakness in this patient were related to dengue disease as the symptoms improved along with the resolving dengue, but the mechanism could be associated with virus neurotropic effect, systemic infection effect or immune mediated.
\end{abstract}

Keywords: Dengue; Myasthenia gravis; Muscle weakness

\section{Introduction}

Dengue is a mosquito-borne viral disease caused by one of four closely related Dengue Virus (DENV) serotypes. It is the second most common mosquito-borne disease affecting human beings after malaria. Around 4 billion people are at risk of the disease, with about 100 million cases of symptomatic dengue occurring annually [1]. In United Kingdom, the prevalene of Myasthenia Gravis (MG) is 15 per 100,000 population [2]. Since the end of the 1990s, evidence of DENV neurotropism has increased [3,4]. Dengue infection can cause neuromuscular complication which can manifest as muscle weakness. Muscle weakness can be found in forms of myalgia, myositis to rhabdomyolysis [5], Guillain-Barre syndrome [6,7] and hypokalemia $[8,9]$.

Pathogenesis of neuromuscular complications and role of host and virus are not clear, and might be associated with virus neurotropic effect, systemic infection effect or immune mediated such as in Guillain - Barre syndrome in dengue infection. In MG, an autoimmune disease affecting the neuromuscular junction, anti acetylcholine receptor (AChR) antibodies caused lysis - mediated by complement, cross - link, decreased production and direct agonist inhibition of AChR [9]. In this case report, a 36 year-old female MG patient, whose symptoms worsen when she suffered from dengue infection. The MG symptoms improved along with resolving dengue infection. There was a possibility that muscle weakness in this patient were related to dengue disease as the symptoms improved along with the resolving dengue, but the mechanism was not clear.

\section{Case Illustration}

Female, 36 year-old was admitted with fever since 4 days prior. The fever was continuously high with no chill. She also complained of arthralgia, headache and extremity muscle weakness. There was no epistaxis or gum bleeding. She complained of difficulty in swallowing food and liquid, difficulty to open eyelids and increasing difficulty to breathe since prior 2 weeks. She did not have history of asthma. Neurological examination found extremity motor strength of 4444 on four extremities, decreased physiological reflexes, 5th and 10th cranial nerve palsy, and ptosis.

She had experienced same complains 8 month ago when she was diagnosed of MG. Her chest computed tomography scan then was normal. She was on pyridostigmine $60 \mathrm{mg}$ three times daily since 8 month ago. Her laboratory examination revealed thrombocytopenia $(93,000 / \mu \mathrm{L})$, positive dengue IgG and IgM using Panbio Dengue IgG IgM Capture ELISA. She received fluid therapy and pyridostigmine 60 mg four times daily. Her complains (ptosis, cranial nerves palsy and extremity motor strength) and laboratory improved, then she was discharged on the 6th day of admission.

\section{Discussion}

$M G$ is an acquired autoimmune disease affecting the neuromuscular junction, especially the nicotinic AChR which located on the post synaptic endplate membrane. Acetylcholine produced and released normally, but the post synaptic effect decreased due to decreased AChR, which its membrane is occupied by antibodies and complement. Anti-AChR antibodies causing the decreased AChR by lysis-mediated by complement, cross-link, decreased production and direct agonist inhibition [9] (Table 1). 
Page 2 of 2

\begin{tabular}{|l|l|l|l|l|l|}
\hline & $24 / 3 / 2014$ & $26 / 3 / 2014$ & $27 / 3 / 2014$ & $28 / 3 / 2014$ & $29 / 3 / 2014$ \\
\hline Ptosis & $+/+$ & $-/+$ & $-/-$ & $-/-$ & $-/-$ \\
\hline Cranial nerves palsy & + & - & - & - & - \\
\hline Extremity motor strength & $4444 \mid 4444$ & $5555 \mid 5555$ & $5555 \mid 5555$ & $5555 \mid 5555$ & $5555 \mid 5555$ \\
& $4444 \mid 4444$ & $5544 \mid 4455$ & $5555 \mid 5555$ & $5555 \mid 5555$ & $5555 \mid 5555$ \\
\hline Hemoglobin & 13.80 & 12.30 & 12.60 & 12.50 & 12.90 \\
\hline Hematocrit & 39.30 & 36.10 & 37.40 & 35.70 & 37.20 \\
\hline Leucocyte & 3,000 & 3,200 & 4,400 & 5,000 & 5,200 \\
\hline Platelet & 93,000 & 62,000 & 89,000 & 116,000 & 168,000 \\
\hline
\end{tabular}

Table 1: Symptoms, physical examinations and laboratory results of the patient

Characters of MG symptoms are the muscle weakness that is more on specific muscle than generalized weakness, more on proximal than distal muscle, weakness that is less in the morning then more in the evening, progressive on exertion and improved with rest [5]. Worsening of MG symptoms are influenced by many factors including emotional distress, systemic diseases (primarily virus respiratory infection), hypothyroidism or hyperthyroidism, pregnancy, menstrual cycle, fever and medication that influenced neuromuscular transmission [9].

This patient experienced extremity muscle weakness, difficulty in swallowing food and liquid, difficulty in opening eyelids and increasing difficulty breathing 8 months ago. She was diagnosed of MG and her symptoms improved by pyridostigmine therapy $60 \mathrm{mg}$ three times daily. Her symptoms repeated 2 weeks ago and worsen 4 days ago, when she was diagnosed with dengue fever. Her symptoms 2 weeks ago were similar to symptoms 8 month ago, with muscle weakness more on specific muscle than generalized weakness, weakness that is less in the morning then more in the evening, progressive on exertion and improved with rest; symptoms of MG. Dengue has incubation period of 4-7 days (range 3-14 days) [1]. The patient started to experienced MG symptoms since 2 weeks ago, which probably was on the first day of the possible incubation period when DENV was inoculated by mosquito and was about to start replication but not yet spreading to lymphoid organ or causing viremia. Other possibilities were the worsening of MG symptoms 2 weeks ago was due to disease flare-up by emotional distress, fever or medication.

Dengue infection can caused neuromuscular complications, which pathogenesis comprised of 3 categories, i.e. complications caused by DENV neurotropic nature, complications associated with systemic complication of dengue infection and post dengue infection complications. Pathogenesis of neuromuscular complications and role of host and virus are not clear, and might be associated with virus neurotropic effect, systemic infection effect and immune mediated [5].

Neuromuscular complications of dengue infection can manifest in muscle weakness. Muscle weakness in dengue infection can be found in forms of myalgia, myositis that can caused rhabdomyolysis [5],
Guillain - Barre syndrome [6,7] and hypokalemia [8,9]. Guillain Barre syndrome in dengue infection caused an immune response that cross react with peripheral nerve components due to cross-reactive epitopes. The immune response can occur in myelin or peripheral nerve axon. Hypokalemia mechanism in dengue infection might be caused by potassium redistribution into cells or transient kidney tubules abnormalities that excreted potassium into urine [8].

No myalgia or myositis symptoms such as muscleache, or hypokalemia were found in this patient. A possible cause of muscle weakness in this patient were due to Guillain - Barre syndrome or worsening of MG symptoms that were influenced by systemic diseases in this case dengue infection. No further examinations such as level of AChR, dengue serotype, or cytokine level (interleukin, interferon, tumor necrotizing factor) were done. Although this could not be confirmed, there was possibility that muscle weakness in this patient were related to dengue infection as the symptoms improved along with the resolving dengue infection.

\section{References}

1. WHO (2009) Dengue guidelines for diagnosis, treatment, prevention and control. Geneva: World Health Organization.

2. Spillane J, Higham E, Kullmann DM (2012) Myasthenia gravis. BMJ 21: $345-349$

3. Solomon T, Dung NM, Vaughn DW, Kneen R, Thao LT, et al. (2000) Neurological manifestations of dengue infection. Lancet 355: 1053-1059.

4. Domingues RB, Kuster GW, Onuki-Castro FL, Souza VA, Levi JE, et al. (2008) Involvement of the central nervous system in patients with dengue virus infection. J Neurol Sci 267: 36-40.

5. Carod-Artal FJ, Wichmann O, Farrar J, Gascón J (2013) Neurological complications of dengue virus infection. Lancet Neurol 12: 906-919.

6. Hahn AF (1998) Guillain-Barré syndrome. Lancet 352: 635-641.

7. Jha S, Ansari MK (2010) Dengue infection causing acute hypokalemic quadriparesis. Neurol India 58: 592-594.

8. Hira HS, Kaur A, Shukla A (2012) Acute neuromuscular weakness associated with dengue infection. J Neurosci Rural Pract 3: 36-39.

9. Khadilkar SV, Sahni AO, Patil SG (2004) Myasthenia gravis. J Assoc Physicians India 52: 897-904. 